\title{
Les organes artificiels
}

Thibaut Raguin ${ }^{1}$, Agnès Dupret-Bories², Christian Debry ${ }^{1}$

> Depuis longtemps, la recherche vise à suppléer les défaillances viscérales et la pénurie de dons par des organes artificiels. Grâce aux nouvelles technologies, à l'ingénierie tissulaire et à la médecine régénérative, de nombreux organes peuvent bénéficier d'un équivalent artificiel: certaines personnes aveugles visualisent des stimulus grâce à des implants rétiniens, I'insuffisant cardiaque peut attendre une greffe grâce à un cœur artificiel, les patients laryngectomisés ont une vie presque normale grâce à un larynx artificiel, et le diabétique, grâce à une pompe sous-cutanée, peut avoir une autorégulation glycémique contrôlée par appareil mobile (smartphone). Les dispositifs de dialyse et d'oxygénation pour les insuffisances respiratoires terminales deviennent portables. De belles perspectives sont en cours d'étude ou pourraient voir le jour dans un avenir proche, mais le recul pour évaluer les effets secondaires n'est pas encore suffisant et le coût de ces nouveaux dispositifs n'est pas négligeable, même si l'avènement des imprimantes trois dimensions peut en réduire les montants. <

Pendant de nombreuses années, la science-fiction a exploré l'idée de traiter les maladies au moyen de dispositifs artificiels. Les références visant à remplacer des organes humains par des dispositifs mécaniques implantables sont présentes dans de nombreux ouvrages littéraires et films: de Robocop à Iron Man, en passant par Frankenstein ou Edward aux mains d'argent, l'homme bionique a toujours été un sujet majeur d'inspiration des auteurs. Avec le vieillissement de la population, une personne de plus de 65 ans sur cinq bénéficie déjà d'un remplacement d'organe (prothèse, implant dentaire, valve, etc.).

Depuis 50 ans, la recherche sur les organes artificiels n'a cessé de progresser, offrant une alternative réelle à la transplantation et une meilleure qualité de vie aux

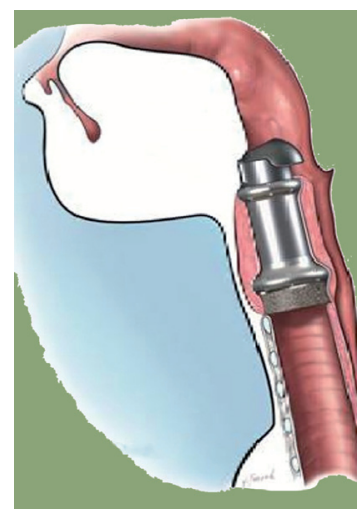

${ }^{1}$ Service ORL et chirurgie cervico-faciale, CHU de Strasbourg, 1, avenue Molière, 67098 Strasbourg Cedex, France. ${ }^{2}$ Service d'otorhinolaryngologie et chirurgie cervico-faciale, Institut Universitaire du Cancer, avenue Hubert Curien, 31100 Toulouse, France. thibaut.raguin@chru-strasbourg.fr

patients. Historiquement, cette recherche a débuté par la «supplémentation » de défaillance de certains organes par un dispositif pouvant remplacer partiellement leur fonction. La première dialyse rénale date de 1945, la pose du premier implant cochléaire (destiné aux déficients auditifs) des années 1960, et le premier pacemaker a été implanté au milieu des années 1970 [1]. Le remplacement d'un organe biologique dans son intégralité par une pièce artificielle est plus récent. Les progrès réalisés en médecine et en bio-ingénierie depuis la fin $d u x x^{e}$ siècle ont permis progressivement le passage de la fiction à la réalité. Même si la solution optimale demeure encore la transplantation d'organes de donneurs apparentés, en cas de pénurie de dons, les organes artificiels représentent un atout majeur pour de très nombreux patients.

De nombreux journaux scientifiques se sont spécialisés dans le domaine des organes artificiels, dont maintenant six revues indexées : Artificial Organs ; Journal of Artificial Organs ; Artificial Organs Today ; Biomaterials, Artificial Cells and Artificial Organs;Biomaterials, Medical Devices and Artificial Organs; et Trends in Biomaterials and Artificial Organs. Une simple recherche effectuée dans la base de données pubmed référencie, en moyenne, plus de 2 publications par jour depuis le début de l'année 2016.

Cet intérêt croissant est lié à une meilleure biocompatibilité des matériaux ainsi qu'une miniaturisation accrue des appareils. De nombreux brevets sur de nouveaux dispositifs sont déposés régulièrement grâce à la recherche réalisée par des sociétés privées le plus souvent en association avec des départements universitaires et les instituts de recherche. L'intérêt que suscitent ces nouvelles technologies offre des perspectives originales pour les professionnels spécialisés, que ce soit les médecins, les ingénieurs, les biologistes ou les bio-ingénieurs. Les prothèses représentent ainsi d'énormes enjeux en termes de santé et d'économie. Le marché mondial des biomatériaux est estimé à plusieurs dizaines de milliards d'euros, une valeur en constante hausse en raison des augmentations de la population et de la durée de vie. 
Parmi les organes qui peuvent être remplacés, la recherche progresse notamment sur le développement d'un cœur ou d'un larynx artificiel, d'un œil bionique, sur le remplacement de rein par des machines «portables », et sur le développement de dispositifs assurant la fonction endocrine du pancréas ou la supplémentation des défaillances respiratoires. L'émergence des imprimantes 3D (3 dimensions) pour remplacer les os ou les vaisseaux est également en plein essor [35] $(\rightarrow)$.

$\rightarrow$ Voir la Synthèse de J.C. Fricain et al., page 52 de ce numéro Le développement et l'accès de plus en plus facile à ces imprimantes 3D ouvrent en effet un nouveau champ de perspective en ingénierie biomédicale: plusieurs sociétés françaises travaillent sur la création d'implants biocéramiques sur mesure à partir d'une conception informatique. Ces implants permettent de compenser des pertes osseuses cranio-maxillo-faciales, dentaires et orthopédiques. Dans le même domaine l'étude, la conception de néo-vaisseaux artificiels construits par impression tridimensionnelle ou d'organes comme la trachée avec implantation de cellules souches est aussi en cours d'évaluation [2, 3].

\section{L'œil}

Selon l'Organisation mondiale de la santé, 314 millions de personnes seraient malvoyantes dans le monde [4]. Parmi ces individus, seule une minorité (environ 45 millions) est effectivement considérée comme profondément aveugle (défini par une acuité visuelle corrigée inférieure à 20/400 sur l'échelle de Snellen ${ }^{1}$ ). La plus grande partie de ces personnes vit dans des pays en développement et la majorité des causes de cécité sont en fait évitables et/ou traitables. La restauration d'une vision fonctionnelle grâce à une prothèse visuelle ne ciblera donc qu'une partie restreinte de la population aveugle. Les prothèses visuelles en cours de conception visent des personnes ayant une perte de la vision profonde mais ayant eu un développement visuel normal (par opposition aux personnes atteintes de cécité congénitale).

La prothèse visuelle se fonde sur les mêmes principes fondamentaux que l'implant cochléaire, élaboré par Marbach et al. en 1982 [5] : les neuroprothèses ciblent et contrôlent la stimulation électrique des nerfs afin de restaurer la fonction perdue. De ce principe sont aussi nés les membres artificiels sophistiqués permettant de rétablir la marche ou la préhension pour les amputés $[6,7]$. D’une manière générale, le principe de fonctionnement d'une neuroprothèse visuelle consiste à remplacer artificiellement la fonction des neurones endommagés. Les images sont captées par une caméra située dans des lunettes portées par le patient puis transmises à un microprocesseur qui les traite et les transmet à l'implant par l'intermédiaire d'une connexion sans fil. L'implant permet une stimulation micro-électrique d'un tableau de minuscules microélectrodes permettant de susciter la perception de modèles organisés de lumière appelés phosphènes [8]. Ces stimulus recréent la perception de formes lumineuses que le patient doit ensuite apprendre à réinterpréter. À ce jour, cette technologie permet une représentation visuelle spatiale mais elle ne traite pas encore des éléments plus complexes tels que les couleurs, les mouvements ou les

${ }^{1}$ Test d'acuité visuelle impliquant la lecture de lettres de différentes tailles.

formes. Le principal défi réside dans la stimulation la plus précise possible de chaque élément sensoriel, afin d'améliorer la sensibilité globale de ces implants [9]. De par leur conception, ces prothèses oculaires sont adaptées à des pathologies dans lesquelles la capture de l'information lumineuse est atteinte mais non la conduction de l'information visuelle et/ou le traitement cortical. C'est le cas notamment de deux pathologies fréquentes, la rétinite pigmentaire ${ }^{2}(R P)$ et la dégénérescence maculaire liée à l'âge ${ }^{3}$ (DMLA), dans lesquelles il y a effectivement une atteinte des cellules photoréceptrices de l'œil causant une perte de vision allant jusqu'à la cécité profonde, la plupart des autres éléments constituant l'œil étant relativement préservés (notamment les cellules neuronales formant le nerf optique) [10-12].

Deux zones d'implantation de la prothèse sont en cours d'étude: une approche sous-rétinienne [33] $(\rightarrow)$ pour laquelle l'implant est placé dans la région des photorécepteurs, et

$(\rightarrow)$ Voir la Nouvelle de $\mathrm{H}$. Lorach, $\mathrm{m} / \mathrm{s}$ $n^{\circ} 10$, octobre 2015 , page 830

une approche épi-rétinienne, pour laquelle l'implant est fixé sur la surface interne de la rétine, à proximité des cellules ganglionnaires ${ }^{4}$. Cette dernière technique a déjà été utilisée en Europe et notamment en France. Un exemple de prothèse de ce type est le système de prothèse rétinienne Argus II, commercialisé par les laboratoires américains Second Sight, destiné à stimuler électriquement la rétine afin de déclencher une perception visuelle chez les personnes aveugles (Figure 1) [13]. L'implant est une prothèse épi-rétinienne implantée par voie chirurgicale à l'intérieur et autour de l'œil. Il comprend une antenne, un boîtier électronique et un faisceau d'électrodes. La vidéo, acquise par une petite caméra fixée sur des lunettes, est envoyée à un petit ordinateur porté par le patient, qui la traite et la transmet à l'implant par le biais d'une connexion sans fil. Les signaux sont ensuite envoyés au faisceau d'électrodes qui émet de petites impulsions électriques. Ces impulsions contournent les photorécepteurs endommagés et stimulent les cellules de la rétine restées indemnes. Transmises le long du nerf optique, ces informations créent la perception de formes lumineuses que le patient doit ensuite apprendre à réinterpréter : cet œil bionique ne permet pas de rétablir une vision

\footnotetext{
${ }^{2}$ Maladie génétique dégénérative de l'œil affectant les photorécepteurs de la rétine et évoluant jusqu'à la cécité.

${ }^{3}$ Maladie dégénérative touchant la partie centrale de la rétine (macula) se déclarant généralement à partir de 50 ans.

${ }^{4}$ Pour rappel, la rétine est une couche de photorécepteurs tapissant l'intérieur du globe oculaire. L'information visuelle captée par ces récepteurs est ensuite transmise à d'autres cellules parmi lesquelles les cellules ganglionnaires, dont les axones forment le nerf optique.
} 

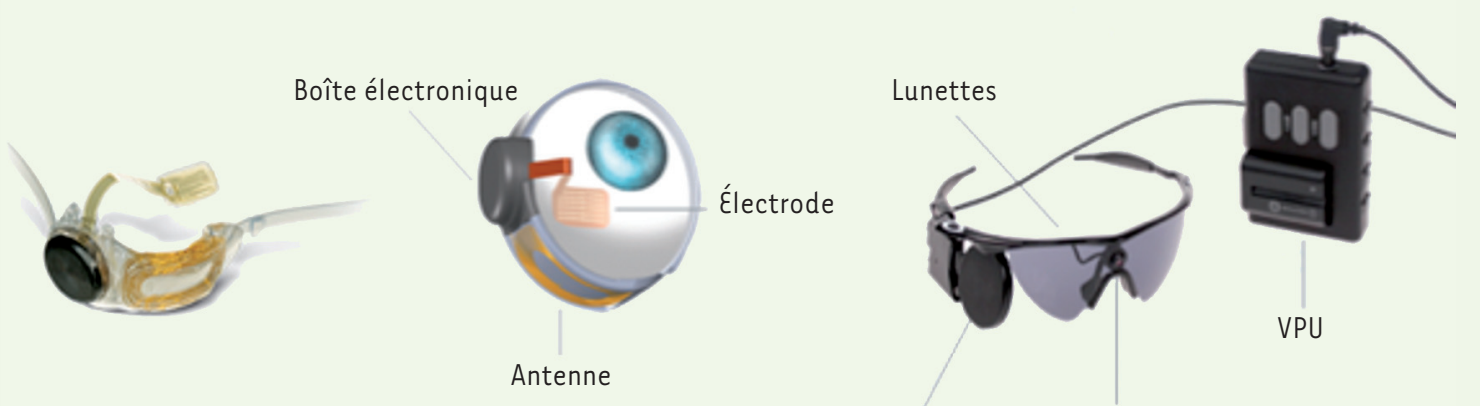

Antenne

Caméra

Figure 1. Implant épi-rétinien et système de recueil de données fixé aux lunettes de la société Second Sight. VPU : video processing unit (d'après [13]).

normale mais construit une image artificielle à partir de laquelle les patients peuvent extraire de l'information visuelle en se fondant sur leur perception des contrastes. Le champ visuel est cependant réduit, et le mouvement oculaire insuffisant pour bénéficier d'un champ visuel correct ; les patients doivent donc bouger la tête pour récupérer l'information visuelle dans leur environnement.

La conception de prothèse visuelle sous-rétinienne est actuellement à l'étude par le Boston Projet Retinal Implant (un grand projet collaboratif comprenant de nombreuses institutions américaines) [14]. Cette approche offre des avantages par rapport aux dispositifs épirétiniens: un risque réduit d'infection oculaire, une diminution de l'érosion à travers les tissus délicats qui couvrent la surface antérieure de l'œil, et la possibilité de placer les électrodes de stimulation plus près des cellules nerveuses de la rétine, améliorant ainsi la sécurité du dispositif et le résultat visuel [15]. La plus grande partie du matériel électronique est située à l'extérieur de l'œil, ce qui évite les complications liées à la production de chaleur et à la corrosion du matériel (Figure 2). Son fonctionnement est semblable à celui de l'implant épirétinien : une caméra miniature montée sur une paire de lunettes est utilisée pour la capture d'image. Ces images sont ensuite analysées par un microprocesseur portatif utilisé pour convertir les données d'image en signal électronique transféré à l'implant, sans fil. Le signal résultant est transmis à la matrice de microélectrodes sous-rétinienne pour stimuler les éléments neuronaux rétiniens survivants.

\section{Le cœur}

Les recherches portant sur le développement d'un cœur artificiel ont été initiées dans les années 1950. Ces études ont été menées pour faire face à la pénurie de dons d'organes et à l'augmentation des pathologies liées notamment au vieillissement de la population comme l'insuffisance cardiaque. Aux États-Unis, près de 5 millions de personnes souffrent d'insuffisance cardiaque et la défaillance de cet organe entraîne 300000 décès par an, pour un coût annuel de 38 milliards de dollars. On estime que 15 à $25 \%$ des patients en insuffisance sont en attente de transplantation [16]. Deux médecins américains, William Kolff and Michael DeBakey, ont été les pionniers du déve- loppement du cœur artificiel dans les années 1960. II existe aujourd'hui plusieurs dispositifs différents, de forme et de structures variables, connus sous le nom VAD (aides ventriculaires périphériques), qui peuvent être implantés (Figure 3). La plupart est développée par des sociétés américaines (DeBakey DAV de MicroMed, HeartMate II de Thoratec, DuraHeart de Terumo, HeartWare HVAD de HeartWare), mais aussi européennes (Incor de Berlin Heart et la société française CARMAT [34]) $(\rightarrow)$.

Ces dispositifs sont, pour la majorité, temporaires: ils assurent la survie des patients en attente d'une greffe cardiaque et constituent donc plutôt un «pont» pour une transplantation. La conception d'un cœur artificiel représente un défi technologique de taille. Le dispositif doit notamment ajuster la circulation sanguine aux besoins de l'organisme tout en conservant une activité anticoagulante efficace. Sa taille est également un facteur important pour s'astreindre de critères morphologiques préimplantatoires. Il faut aussi assurer son alimentation en énergie; à ce niveau, les progrès sont réels: les systèmes les plus récents fonctionnent par induction (transmission d'énergie sans contact mécanique ou électrique), si bien que la cage thoracique n'abrite plus de fils électriques. Parmi les dispositifs actuels, quelques-uns offrent la possibilité aux patients de retrouver une réelle mobilité et une autonomie sans nécessité d'hospitalisation continue. Ces dispositifs artificiels permettent d'assurer une transition jusqu'à la transplantation et de renforcer les fonctions cardiaques pendant de nombreux mois.

Les cœurs artificiels actuels ont encore quelques limites et risques, essentiellement d'infections et d'atteintes thrombo-emboliques (formation de caillots sanguins dans les vaisseaux) liés à des mécanismes qui ont été 

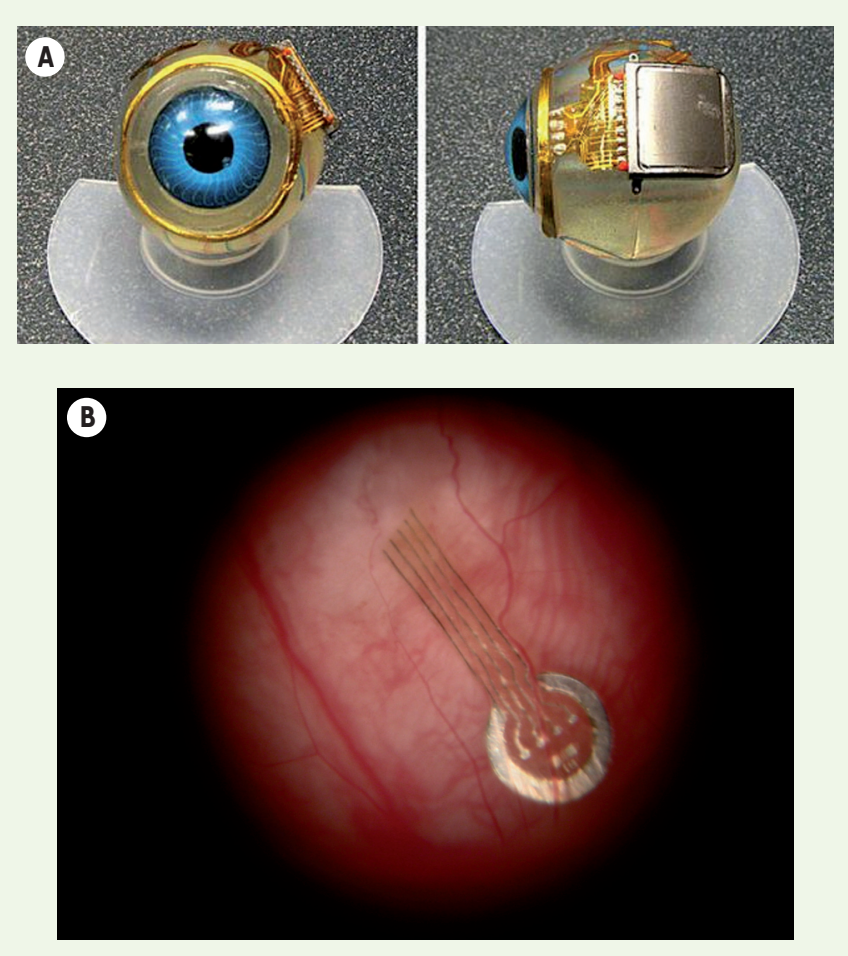

Figure 2. Vue de l'implant sous-rétinien avant (A) et après pose chirurgicale (B). Boston Retinal Implant Project and Bionic Eye Technologies Inc.

identifiés [17], mais ils présentent aussi d'autres effets secondaires qui sont en cours d'évaluation: selon le dispositif implanté, l'effet pulsatile du flux sanguin généré par le cœur artificiel est variable et l'absence de pulsatilité au long cours n'a pas encore été déterminée (certains dispositifs sont à flux continu et ne reproduisent donc plus le pouls) [18-20]. Pour les dispositifs pulsatiles, d'autres risques sont identifiés, comme la rupture de membrane ou une défaillance de soupape.

La conception des nouveaux cœurs artificiels affiche donc plusieurs objectifs :

- Étendre l'éventail de patients pouvant bénéficier de cette technologie, avec notamment un développement pédiatrique. Pour cela, de nouveaux matériaux de construction sont à l'étude avec des composants plus légers, plus sûrs et plus facilement portables, afin d'obtenir des dispositifs petits et compacts [22, 23].

- Résoudre les problèmes de consommation d'énergie, défi majeur encore mal maîtrisé, par la mise en œuvre de nouveaux systèmes d'alimentation basés sur la technologie sans fil, sur des systèmes d'induction, ou des systèmes utilisant des champs magnétiques à haute intensité [24].

- Réduire les risques d'accident vasculaire cérébral hémorragique et d'événements thrombo-emboliques en utilisant des valves tissulaires biologiques et des matériaux spécifiques peu thrombogènes, qui pourraient être incorporés dans les sites en contact prolongé avec le sang.
- Améliorer la surveillance des dispositifs grâce à la télémédecine et l'utilisation de capteurs externes fonctionnant en réseau non filaire comme la WiFi.

L'incorporation de ces innovations dans les prochaines générations de dispositifs permettra d'améliorer la tolérance des systèmes et la prise en charge clinique globale des patients atteints de défaillance cardiaque en stade terminal.

\section{Les poumons}

Bien que l'oxygénation par des machines externes (oxygénation par membrane extracorporelle, ECMO) soit un moyen de remplacement bien connu de la fonction pulmonaire, il n'existe à l'heure actuelle aucun prototype de poumons artificiels qui pourrait être implantable. Le principal obstacle et défi pour un tel dispositif sont la fixation du poumon artificiel au bloc cardiaque et la mise en place des échanges gazeux intravasculaires. Différents systèmes sont à l'étude, comme le système IVOX pour lequel l'échange gazeux s'effectue grâce à un cathéter placé dans la veine cave. Des dispositifs de plus petites tailles (comme le système Cardiohelp, une ECMO portable) sont aussi en cours de développement $[25,26]$.

L'équipe du Pr Mercier (Centre chirurgical Marie-Lannelongue, Le Plessis Robinson) a bénéficié d'une subvention ministérielle (programme d'investissements d'avenir de l'Agence nationale de la recherche, BIOART-LUNG 2020) pour réaliser un poumon artificiel extracorporel pour le traitement de patients souffrant d'une hypertension artérielle pulmonaire qui ne peuvent pas bénéficier d'une transplantation. Dans ce dispositif, les deux poumons malades du patient seront laissés en place, le «troisième » sera fixé à l'extérieur du corps, au niveau du thorax par une ceinture. Sur le même principe qu'une dialyse rénale, ce poumon portatif permettra de remplacer l'activité pulmonaire: grâce à une canule placée au niveau de la veine jugulaire ou dans la veine sous-clavière, et rejoignant la partie droite du cœur, le sang veineux passera dans la machine afin de l'oxygéner et d'éliminer le dioxyde de carbone. Le sang sera réinjecté dans la partie gauche du cœur pour être distribué aux organes. Le but de ce poumon artificiel, qui court-circuite totalement les poumons malades, est donc d'être portatif et le plus autonome possible en matière d'énergie pour pouvoir espérer un bénéfice extrahospitalier. L'un des plus grands défis de ce projet est donc le développement d'une batterie légère et suffisamment autonome pour être transportée facilement et longtemps, les patients nécessitant cette machine nuit et jour. II est prévu de tester cette innovation chez l'homme en décembre 2020. 
$\varepsilon n$ parallèle, les recherches en médecine régénérative et ingénierie tissulaire pourront également permettre d'accélérer le développement de thérapies et de pallier les insuffisances respiratoires terminales en attente de greffe [27].

\section{Le pancréas}

Les progrès récents concernant les médicaments antidiabétiques, les pompes à insuline, les systèmes de surveillance continue de la glycémie et les moyens informatiques de contrôle, ont entraîné une accélération des innovations dans le développement de dispositifs de pancréas artificiel. Ces dispositifs sont de deux types: les systèmes dits externes et les systèmes dits internes.

Les systèmes externes permettent l'administration sous-cutanée de médicaments. Il existe deux grandes approches pour obtenir un contrôle en boucle fermée de la glycémie chez les personnes diabétiques: (1) l'approche unihormonale qui utilise l'insuline pour réduire la glycémie et repose sur des analyses informatiques complexes pour réduire le risque d'hypoglycémie et (2) l'approche bihormonale qui utilise à la fois l'insuline pour abaisser la glycémie et le glucagon pour l'élever, et qui repose également sur des algorithmes complexes pour assurer la sécurité de l'utilisateur.

Les appareils internes sont constitués d'un système de microprocesseurs qui mesurent les niveaux de glucose dans le sang et répondent automatiquement par des perfusions d'insuline. Aucun modèle implantable n'est actuellement disponible dans le commerce, mais la réduction progressive du volume et l'augmentation de la précision de ces dispositifs indiquent que, dans un proche avenir, un «pancréas endocrine artificiel implantable » peut devenir disponible pour traiter le diabète [28].

Les progrès de la recherche sur le pancréas artificiel depuis le début du siècle ont été possibles grâce à la révolution qu'ont apporté l'ordinateur et la miniaturisation de la technologie électronique. L'avènement des smartphones modernes (ou ordiphones) a ainsi rendu possible l'utilisation de cette technologie comme pièce maîtresse de l'ingénierie d'un pancréas artificiel. Grâce à ces avancées, un pancréas artificiel ou bionique semble être envisageable à court terme.

\section{Les reins}

La dialyse est la forme la plus largement utilisée de remplacement de la fonction d'un organe interne par un dispositif artificiel. Cependant, les séances de dialyse, durant lesquelles le patient reste littéralement prisonnier de la machine, ont un impact majeur sur la qualité de vie des personnes atteintes d'insuffisance rénale. La miniaturisation des systèmes de dialyse a maintenant permis de construire et, à titre expérimental, d'utiliser des dispositifs portables qui peuvent être fixés au corps du patient, lui permettant ainsi de réaliser certaines activités pendant la période de dialyse. En 2009, Gura et al. ont publié un document sur un dispositif non-implantable de dialyse qui a été adapté pour être inséré dans un gilet permettant ainsi au patient une mobilité totale lors de ses séances de dialyse [29].

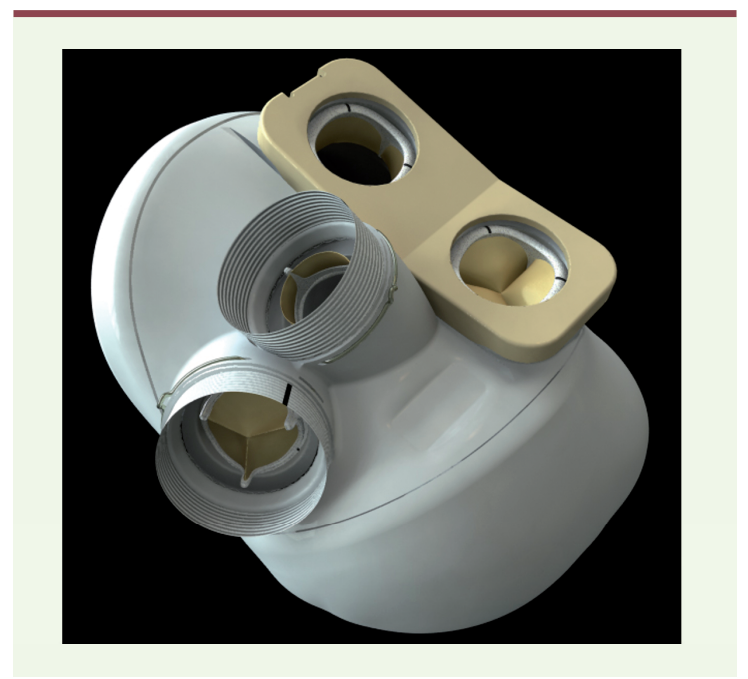

Figure 3. Cour artificiel de la société CARMAT.

En parallèle, les chercheurs travaillent depuis longtemps sur le développement de reins artificiels implantables. Plusieurs dispositifs de ce type sont en phase de développement. En 2010, des chercheurs américains ont réussi à implanter un rein artificiel chez l'animal : constitué d'un système de filtration et d'un bioréacteur conçu avec du tissu rénal biologique, il ne nécessite aucune source d'énergie externe. II faudra cependant encore des années pour que ce type de dispositif puisse être utilisé dans la pratique clinique [30].

\section{Le larynx}

Les cancers des voies aéro-digestives supérieures sont placés au sixième rang des cancers dans le monde, le carcinome épidermoïde ${ }^{5}$ étant le type histologique le plus fréquent [31]. Quand ce cancer est étendu à tout le larynx, une opération chirurgicale de type laryngectomie totale est nécessaire. Entre 1500 et 1600 laryngectomies totales sont effectuées chaque année en France. Cette opération est assez mutilante, empêchant la phonation (les cordes vocales sont ablatées avec le larynx malade) et nécessitant un trachéostome (abouchement de la trachée au niveau du sternum) pour assurer la respiration.

Les laboratoires Protipmédical et le service d'otorhino-laryngologie de Strasbourg ont créé un larynx artificiel en titane, biomatériau retenu pour sa résistance mais surtout pour son acceptabilité par le corps humain. Ce larynx est constitué d'un alliage de titane solide et de titane poreux permettant une

\footnotetext{
${ }^{5}$ Tumeur de l'épithélium malpighien pouvant toucher de nombreux organes.
} 
meilleure colonisation cellulaire et donc moins de rejet. Vingt années de recherche ont été nécessaires pour développer cette prothèse. L'implantation se réalise en deux étapes. La première vise à enlever la tumeur (laryngectomie totale) et à mettre en place une bague en titane reliant la trachée à la base de la langue. Une valve mécanique est ensuite placée au niveau de la partie haute de la bague pour rendre l'ensemble fonctionnel et permettre de diriger l'air vers la trachée et les liquides et solides vers l'œsophage. Le patient peut donc respirer normalement et déglutir sans fausse route (Figure 4). Seule la phonation est modifiée, la voix est plutôt chuchotée [32]. L'objectif final est donc d'améliorer la qualité de vie des patients laryngectomisés en leur permettant, après rééducation orthophonique, d'avoir une vie presque identique à celle précédant l'intervention. Cependant les implantations actuelles sont encore en cours d'essai et, même si le patient peut respirer de manière physiologique au travers de la prothèse, il conserve toujours une trachéotomie de sureté. Une dizaine de patients ont déjà bénéficié de ces prothèses avec un recul de plus de 14 mois pour certains.

\section{Conclusion}

La médecine et l'ingénierie avancent de manière conjointe dans le développement d'organes artificiels. Des études récentes, comme celles décrites dans cette revue, montrent que nous aurons bientôt de nouvelles alternatives thérapeutiques disponibles. Cependant, l'impact réel de ces dispositifs en matière de longévité et de retentissement sur la qualité de vie au sein de la population reste inconnu. Le coût, encore estimatif mais déjà élevé, de ces produits et de leur utilisation est aussi à prendre en compte dans le contexte d'une médecine moderne déjà très coûteuse, même si l'avènement des imprimantes 3D permettra sans doute de la réduire. Des approches très intéressantes sont en cours d'étude ou pourraient voir le jour dans un avenir proche. Cependant, leurs possibles effets secondaires ne sont pas encore évalués, ce qui ne permet donc pas encore de généraliser l'utilisation de ces organes artificiels. $\diamond$

\section{SUMMARY}

\section{Artificial organs}

Research has been fighting against organ failure and shortage of donations by supplying artificial organs for many years. With the raise of new technologies, tissue engineering and regenerative medicine, many organs can benefit of an artificial equivalent: thanks to retinal implants some blind people can visualize stimuli, an artificial heart can be proposed in case of cardiac failure while awaiting for a heart transplant, artificial larynx enables laryngectomy patients to an almost normal life, while the diabetic can get a glycemic self-regulation controlled by smartphones with an artificial device. Dialysis devices become portable, as well as the oxygenation systems for terminal respiratory failure. Bright prospects are being explored or might emerge in a near future. However, the retrospective assessment of putative side effects is not yet sufficient. Finally, the cost of these new devices is significant even if the advent of three dimensional printers may reduce it. $\diamond$

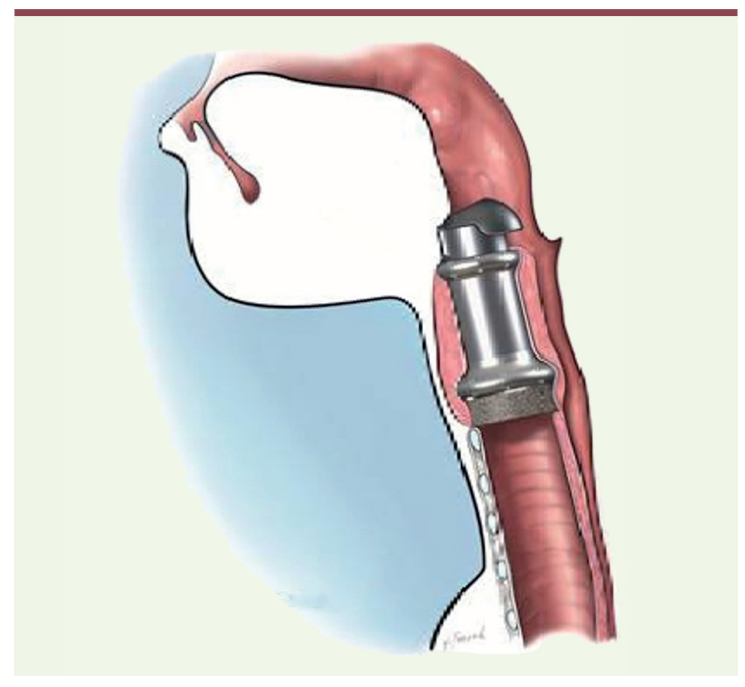

Figure 4. Larynx artificiel de la société Protip Medical en place avec la bague et la valve mécanique.

\section{LIENS D'INTÉRÊT}

Le Professeur C. Debry est actionnaire de la société Protip Medical. T. Raguin et A. Dupret-Bories déclarent n'avoir aucun lien d'intérêt concernant les données publiées dans cet article.

\section{RÉFÉRENCES}

1. Blakeslee S. Willem Kolff, doctor who invented kidney and heart machines, dies at 97. The New York Times 2009. http://www.nytimes.com/2009/02/13/ health/13kolff.html

2. Pati F, Jang J, $\mathrm{Ha} \mathrm{DH}$, et al. Printing three-dimensional tissue analogues with decellularized extracellular matrix bioink. Nat Commun $2014 ; 5$ : 3935 .

3. Lee VK, Dai G. Printing of three-dimensional tissue analogs for regenerative medicine. Ann Biomed Eng 2016 ; April 11. doi : 10.1007/ s10439-016-1613-7.

4. WHO. Visual impairment and blindness. http://www.who.int/mediacentre/ factsheets/fs282/en/

5. Marbach WD, Zabarsky M, Hoban P, Nelson C. Building the bionic man. Newsweek $1982 ; 100: 78-9$.

6. Laferrier JZ, Gailey R. Advances in lower-limb prosthetic technology. Phys Med Rehabil Clin North Am $2010 ; 21: 87-110$.

7. Craelius W. The bionic man: restoring mobility. Science 2002 ; 295 : 1018-21.

8. Peterman MC, Bloom DM, Lee $C$, et al. Localized neurotransmitter release for use in a prototype retinal interface. Invest Ophthalmol Vis Sci $2003 ; 44$ : 3144-9.

9. Dagnelie G, Schuchard RA. The state of visual prosthetics: hype or promise? J Rehabil Res Dev 2007 ; 44 : XI-XIV.

10. Bunker CH, Berson EL, Bromley WC, et al. Prevalence of retinitis pigmentosa in Maine. Am J Ophthalmol $1984 ; 97: 357-65$.

11. Klein R, Klein BE, Jensen SC, Meuer SM. The five-year incidence and progression of age-related maculopathy: the Beaver Dam Eye Study. Ophthalmology $1997 ; 104: 7-21$.

12. Humayun MS, de Juan $\varepsilon$, Dagnelie G, et al. Visual perception elicited by electrical stimulation of retina in blind humans. Arch Ophthalmol 1996; $114: 40-6$

13. Second Sight Argus ${ }^{\circledR}$ II Retinal Prosthesis System. http://www.secondsight. com/g-the-argus-ii-prosthesis-system-pf-en.html

14. Shire DB, Kelly SK, Chen J, et al. Development and implantation of a minimally invasive wireless subretinal neurostimulator. Iદદદ Trans Biomed Eng 2009; 56 : 2502-11.

15. Javaheri M, Hahn DS, Lakhanpal RR, et al. Retinal prostheses for the blind. Ann Acad Med Singapore 2006 ; 35 : 137-44. 


\section{RÉFÉRENCES}

16. Copeland JG, Smith RG, Arabia FA, et al. Cardiac replacement with a total artificial heart as a bridge to transplantation. $N$ Engl J Med 2004 ; $351: 859-67$.

17. Fox CS, McKenna KL, Allaire PE, et al. Total artificial hearts: past, current, and future. J Card Surg $2015 ; 30: 856-64$

18. Kirklin JK, Naftel DC, Kormos RL, et al. The fourth INTERMACS annual report: 4,000 implants and counting. J Heart Lung Transplant $2012 ; 31$ : 117-26.

19. Cheng A, Williamitis CA, Slaughter MS. Comparison of continuous-flow and pulsatile-flow left ventricular assist devices: is there an advantage to pulsatility? Ann Cardiothorac Surg $2014 ; 3$ : 573-81.

20. Allen GS, Murray KD, Olsen DB. The importance of pulsatile and nonpulsatile flow in the design of blood pumps. Artif Organs $1997 ; 21: 922-8$

21. Zimpfer $D$, Wieselthaler $G$, Czerny $M$, et al. Neurocognitive function in patients with ventricular assist devices: a comparison of pulsatile and continuous blood flow devices. ASAIO J 1992 ; 52 : 24-7.

22. Olsen DB. The history of continuous-flow blood pumps. Artif Organs $2000 ; 24: 401-4$

23. HeartWare. http://ir.heartware.com/phoenix.zhtml?c=187755\&p=irol-newsArticle\&ID=1646139

24. Kassif $y$, Zilbershlag $M$, Levi $M$, et al. A new universal wireless transcutaneous energy transfer (TET) system for implantable LVADs - Preliminary in vitro and in vivo results. J Heart Lung Transplant $2013 ; 32$ : S140-1.

25. Haneya A, Philipp A, Foltan M, et al. First experience with the new portable extracorporeal membrane oxygenation system Cardiohelp for severe respiratory failure in adults. Perfusion 2012 ; $27: 150-5$.

26. Maquet, CARDIOHELP System. http://www.maquet.com/int/products/cardiohelp-system/
27. Martin JT, Zwischenberger JB. Artificial lung and novel devices for respiratory support. Semin Thorac Cardiovasc Surg $2013 ; 25: 70-5$.

28. Peyser T, Dassau $\varepsilon$, Breton M, Skyler JS. The artificial pancreas: current status and future prospects in the management of diabetes. Ann NY Acad Sci $2014 ; 1311: 102-23$

29. Gura V, Ronco C, Davenport A. The wearable artificial kidney, why and how: from holy grail to reality. Semin Dial $2009 ; 22: 13-7$.

30. Kooman JP, Joles JA, Gerritsen KGF. Creating a wearable artificial kidney: where are we now? Expert Rev Med Devices $2015 ; 12$ : 373-6.

31. Jégu J, Binder-Foucard F, Borel C, Velten M. Trends over three decades of the risk of second primary cancer among patients with head and neck cancer. Oral Oncol $2013 ; 49: 9-14$

32. Debry C, Dupret-Bories A, Vrana NE, et al. Laryngeal replacement with an artificial larynx after total laryngectomy: the possibility of restoring larynx functionality in the future. Head Neck $2014 ; 36: 1669-73$

33. Lorach $H$. Prothèses rétiniennes : des implants photovoltaïques à haute résolution. Med Sci (Paris) $2015 ; 31: 830-1$

34. Ménard J. Quelles sont les clés du succès de CARMAT ? Med Sci (Paris) 2014 ; $30: 204-5$.

35. Fricain JC, De Olivera H, Devillard R, et al. Impression 3D en médecine régénératrice et ingénierie tissulaire. Med Sci (Paris) 2017 ; 33 : 52-9.

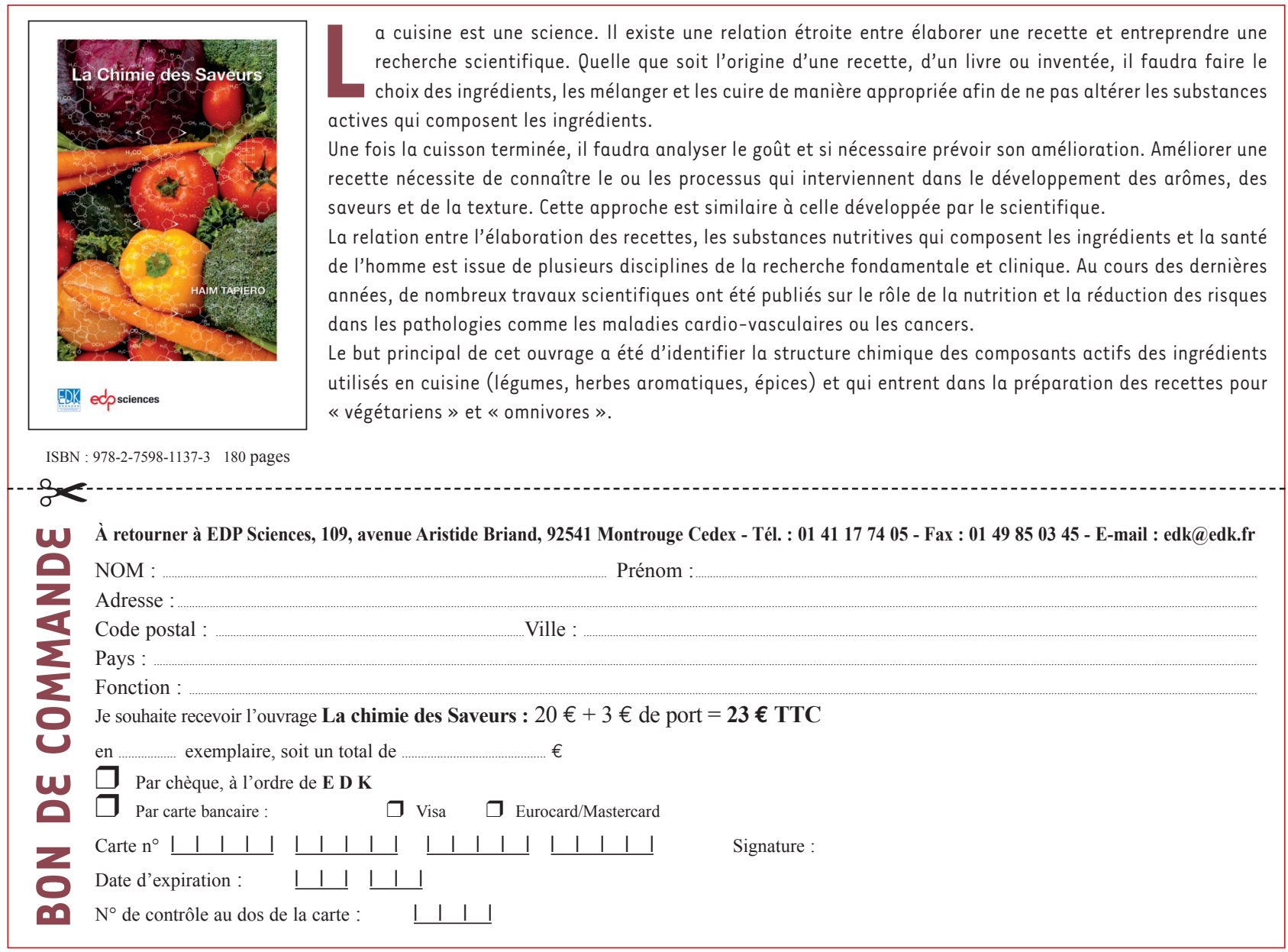

